\title{
Predicting mortality from cervical cancer after negative smear test results
}

\author{
Gerrit J van Oortmarssen, J Dik F Habbema, Marjolein van Ballegooijen
}

\begin{abstract}
Objective-To assess the relative protection against death from cervical cancer after two or more negative smear test results and compare it with the protection against invasive cancer estimated by an International Agency for Research on Cancer (IARC) working group in an analysis of data from 10 large screening programmes.
\end{abstract}

Design-Comparison of risk of death from cervical cancer after two or more negative smear results with the risk in unscreened women by using a model constructed with data from the British Columbia screening programme.

Main outcome measures - Mortality from and incidence of invasive cancer.

Results-In women with two negative smear results estimates of protection against cervical cancer were about $50 \%$ higher when lethal invasive cancer was used as the criterion rather than all invasive cancer. This difference was due to these women being more likely to attend for further tests at which invasive cancer could be detected: screen detected cancer has a better prognosis than clinically diagnosed cancer. Screening intervals could be longer than three years: screening women aged 35-64 every five years was predicted to result in a $\mathbf{9 0 \%}$ reduction in mortality from cervical cancer.

Conclusion-Because protection from mortality is higher than protection from disease and because of the high costs and negative side effects of frequent screening, screening intervals should be longer than three years.

\section{Introduction}

The International Agency for Research on Cancer (IARC) working group on evaluation of cervical cancer screening programmes has analysed data from large screening programmes in Europe and North America.' Protection against cervical cancer after negative smear test results was measured by the risk of invasive cancer. The relative protection - that is, the ratio of the risks in unscreened and screened women-decreases with increasing time since the last smear. Analysis of this decreasing trend may be useful in deciding about the interval between smears. The IARC group concluded that the intervals between screening should be three years or less. ${ }^{2}$

The primary aim of cervical cancer screening is to prevent death from cervical cancer. Using death rather than invasive cancer as the criterion will result in different estimates for the protection after negative smear test results. Women who have had negative smear test results are likely to have further smear tests, and invasive cancers may not only surface clinically but also be detected by these subsequent smears. On average, these invasive cancers detected by screening have a much better prognosis than those that are clinically diagnosed. When comparing women who had one or more negative smear test results with unscreened women, the relative protection against mortality from cervical cancer will therefore be higher than the relative protection against invasive cancer. We investigated the size of the difference between these two relative protection rates, and the implications for recommendations about the screening interval.

\section{Methods}

The aim of screening for cervical cancer is to reduce the risk of diagnosing an invasive cancer that would eventually result in death from the disease. The next screening should therefore be scheduled when the risk of lethal invasive cancer is considered to have become too high. We therefore used protection against lethal invasive cancer as an indicator for protection against mortality.

Information on the survival of patients with invasive cancers was not available in the IARC study. We therefore used a model for progression of the disease which links the risks of lethal invasive cancer to the data on risk of invasive cancer obtained in the IARC study. We constructed a model which has four stages: no cervical cancer, preinvasive cervical cancer, preclinical invasive cervical cancer, and clinical invasive cervical cancer. We used data from the cohort study of cervical cancer screening in British Columbia ${ }^{3}$ to estimate the parameters of this model. ${ }^{4}$ The preinvasive stages dysplasia and carcinoma in situ together had an estimated mean duration of 12.3 (95\% confidence interval 9.7 to 14.7 ) years with a standard deviation of $5.8(4.6$ to $11 \cdot 1)$ years. The estimated mean duration of preclinical invasive stages was five years. The sensitivity of the smear test was estimated at $80 \%$. These values fitted the data from British Columbia well, ${ }^{4}$ and agreed with similar analyses of more recent screening data from the Netherlands ${ }^{5}$ and Sweden. ${ }^{6}$

To be consistent with the IARC study, we included screen detected invasive cancers in the calculations for the model. On the basis of the IARC report we estimated that each year $33 \%$ of women who have already had two or more (negative) smear test results will have another smear test. ${ }^{1}$ We used this estimate in the model, to predict the relative risk of invasive cancer; these predictions agreed with the IARC group finding of $50 \%$ reduction in risk after 6-10 years after (at least) two negative smear test results. The fit of the IARC results could be improved further by increasing the standard deviation of the duration of preinvasive lesions to $7 \cdot 3$ years, which is still well within the confidence limits of the British Columbia estimates. A detailed presentation of this comparison will be given elsewhere. The resulting best fitting model was used to translate the protection against invasive cancer $P_{I}$ into protection against lethal invasive cancer $P_{M}$.

The model gives the proportion of the invasive cancers that are screen detected(s). On the basis of estimates of the average lethalities $\mathrm{q}_{\mathrm{s}}$ and $\mathrm{q}_{\mathrm{c}}$ of screen detected and clinically diagnosed invasive cervica cancers, we used the following formula to calculate the protection against lethal invasive cancer:

$\mathrm{P}_{\mathrm{M}}=\mathrm{P}_{\mathrm{I}}\left((1-\mathrm{s})+\mathrm{s} \times \mathrm{q}_{\mathrm{s}} / \mathrm{q}_{\mathrm{c}}\right)$.

We assumed a relative five year survival rate of $90 \%$ for screen detected invasive cancers, which we derived from combining stage distribution of screen detected
Correspondence to:

BMF 1992;305:449-51 
invasive cancers and stage specific 5 year survival data. ${ }^{?}$ Assuming a $60 \% 5$ year survival for clinically diagnosed cervical cancers gives a 1:4 lethality ratio $\left(\mathrm{q}_{\mathrm{s}} / \mathrm{q}_{\mathrm{c}}=0 \cdot 25\right)$. Some studies have reported survival statistics for clinically diagnosed cancers in the range $65 \%-70 \%,{ }^{7-9}$ but these figures are higher because stage IA and IB cancers detected by screening were included. We assumed that the 1:4 lethality ratio after 5 years of follow up persisted in the long term; we also considered a lethality ratio of $1: 3$

\section{Results}

The figure shows the relative protection against invasive cancer and against lethal invasive cancer predicted by our model for a 45 year old woman who has had two negative smear test results at an interval of three years assuming a 1:4 lethality ratio between screen detected and clinically diagnosed cancers. The points give the relative protection against invasive cervical cancer for 35-64 year old women estimated by the IARC study group, ${ }^{2}$ which we used in our model to produce the dashed curve. The model predicted protection against mortality from cervical cancer after negative smear results was about $50 \%$ higher than the protection against invasive cancer. If a more conservative lethality ratio of $1: 3$ was assumed, the relative protection against mortality from cervical cancer was still about $30-40 \%$ higher than the IARC estimates would suggest.

In the model, the durations of preclinical invasive lesions have an inherent one to one relation with the durations of preinvasive lesions. This assumption implies that fast growing preinvasive lesions will also develop rapidly when they have become invasive. Screening tends to pick up slow growing lesions and to miss fast growing lesions (length biased sampling). Shortly after two negative smear results some women will already have fast growing lesions that may later be diagnosed clinically, resulting in a small proportion of screen detected cancers and a low prediction of the relative protection against lethal invasive cancers (see formula). This effect is strongest in younger age groups. In the model, most cervical cancers occurring at younger ages are assumed to be fast growing lesions that have a lower probability of being detected by regular screening. Thus, the relative protection against lethal invasive cancer in women under age 30 is predicted to be much lower than for women between ages 35 and 65, for whom predictions are similar to those presented in the figure.

We also considered a model with a fixed four year duration of the preclinical invasive stage. The fit for the two data sets (British Columbia and IARC) was as good as with the former model. The length biased sampling phenomenon did not occur in this model, and therefore it predicted that a higher proportion of invasive cancers will be detected by screening. As a result the relative protection against lethal invasive cancer in the first years since the last negative smear test result was almost $100 \%$ higher than the protection against all invasive cancers.

In reality, the development of preclinical invasive cancer will be in between the extreme situations assumed in the two models and the relative protection against lethal invasive cancer will be between $50 \%$ and $100 \%$ higher than for all invasive cancers.

\section{SCREENING FREQUENCIES}

The IARC working group calculated the reduction in cumulative incidence of invasive cervical cancer for different screening frequencies in women aged 35-64. It assumed that all women participate in screening. For example, the figure shows that the incidence of all invasive cancers in the first three years after a second

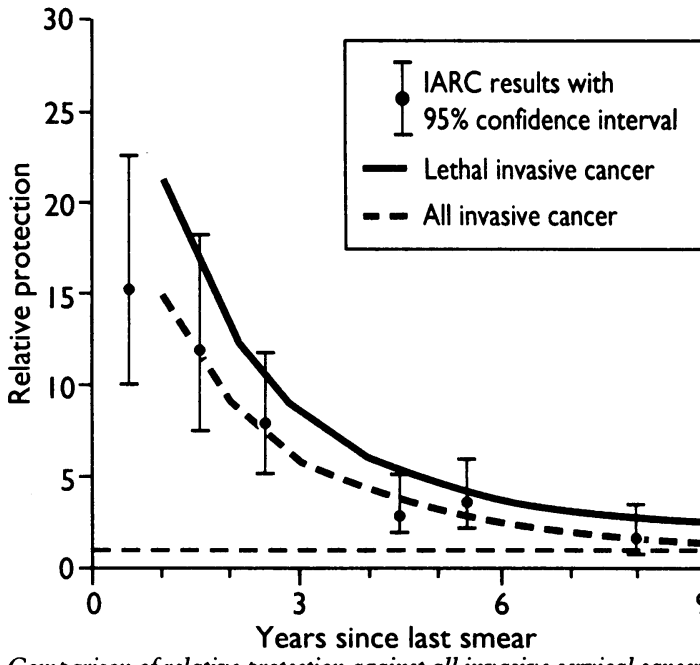

Comparison of relative protection against all invasive cervical cancers as estimated by the IARC group $p^{2}$ with model predictions of protection against lethal invasive and all invasive cervical cancer after two negative smear test results (three years apart) in 45 year old women. It is assumed that each subsequent year $33 \%$ of women who have not yet had a third smear will have one. The lethality ratio of screen detected to clinically diagnosed cancers is assumed to be 1:4.

negative smear test result is between 10 and 11 times lower on average than in a situation without screening, resulting in an estimated $90.4 \%$ reduction in cumulative incidence between the ages 35 and 64 with a three year screening interval. We used the model to derive corresponding figures for the reduction in cumulative incidence of lethal invasive cancer (table). The IARC statement that the screening interval should be three years or less corresponds with a reduction of at least $90 \%$. If this same percentage is applied to reduction in mortality from cervical cancer our model calculations show that an interval of five years is still acceptable. More frequent screening will give little additional benefit. For example, changing from a five year to a three year interval implies that all efforts (taking smears, cytological analysis, follow up, etc) increase by two thirds, whereas the benefits increase by less than $5 \%$, resulting in a 14 times worse marginal cost effectiveness ratio.

Percentage reduction in the cumulative incidence and mortality of cervical cancer in women aged 35-64 for different frequencies of screening ${ }^{\star}$

\begin{tabular}{cccc}
\hline & \multicolumn{3}{c}{$\%$ Reduction in: } \\
\cline { 2 - 4 } $\begin{array}{c}\text { Interval between } \\
\text { screenings (years) }\end{array}$ & $\begin{array}{c}\text { Cumulative } \\
\text { incidence } \\
\text { (A) }\end{array}$ & $\begin{array}{c}\text { Cumulative } \\
\text { incidence } \\
\text { (B) }\end{array}$ & $\begin{array}{c}\text { Cumulative } \\
\text { mortality } \\
\text { (C) }\end{array}$ \\
\hline 1 & 93.5 & 95.4 & 96.9 \\
2 & 92.5 & 93.3 & 95.3 \\
3 & 90.4 & 91.0 & 93.7 \\
4 & $88.4 \dagger$ & 88.3 & 91.8 \\
5 & 83.6 & 85.2 & 89.7 \\
10 & 64.1 & 68.1 & 80.0 \\
\hline
\end{tabular}

${ }^{\star A} \mathrm{~A}$ was calculated by the IARC working group ${ }^{2} ; \mathrm{B}$ and $\mathrm{C}$ are model based predictions.

Calculated from IARC data.

In an earlier analysis we used a more detailed model based on Dutch epidemiological, medical, and economic data to predict the effects and costs of cervical cancer screening policies. ${ }^{5}$ For the Netherlands we calculated a cost effectiveness ratio of about $£ 8000$ per life year gained for a screening policy with five yearly invitations between the ages of 37 and 62 , assuming an average attendance rate of $65 \% .^{510}$ In this model the additional effects of a policy with more frequent invitations (three yearly between ages 36 and 63 ) are higher than in the case with $100 \%$ attendance because of irregular participation patterns. The marginal costs per life year gained with the three yearly policy in comparison with the five yearly policy were 
about $£ 25000$. Whereas the cost per life year gained of $£ 8000$ for a five yearly interval may be considered acceptable, the marginal costs per life year gained by screening every three years are high in comparison with the costs of many other health care facilities. In addition, we found that increasing the screening frequency will worsen the balance between favourable and adverse health effects (unnecessary treatment of women with false positive results and regressive lesions) of screening. ${ }^{.1}$

Our analysis is based on published data from studies that are now more than 10 years old. Reports of an increasing incidence and mortality, especially in younger women, do not influence our results since our estimates are predominantly determined by the duration of preinvasive stages and the sensitivity of the screening test. These factors could also change over time,$^{12}$ stressing the need for continuous re-evaluation of the protection afforded by negative smear test results.

\section{Conclusion}

The most serious negative effects of cervical cancer are early death and the serious morbidity associated with advanced disease. Therefore, reduction of the risk of death from cervical cancer should be the primary criterion in evaluating screening policies. Reduction in incidence of invasive cancer carries an additional benefit since some major therapeutic procedures and their associated morbidity will be avoided, but this should be considered in conjunction with the negative health effects of diagnostic and therapeutic procedures that are induced by screening. ${ }^{.1}$

The use of reduction in incidence as a proxy for reduction in mortality is appealing. However, we have shown that protection against invasive cancer underestimates protection against mortality. The two criteria will lead to different recommendations: screening intervals based on mortality are about $50 \%$ longer than those based on incidence. This difference is caused by the good survival of women with screen detected invasive cancers.

On the basis of our calculations of the reduction in cervical cancer mortality we conclude that screening intervals of five years are appropriate. Regular screening at this interval in the age group 35-64 is expected to give about $90 \%$ reduction in the risk of dying from cervical cancer. More intensive screening will give little additional benefit, and should be discouraged in view of the adverse effects and the high costs.

This study was supported by the Ministry of Welfare, Health, and Cultural Affairs in the Netherlands. We thank Drs A B Miller and D A Boyes for help in analysing data from British Columbia.

1 Hakama M, Miller AB, Day NE, eds. Screening for cancer of the uterine cervix. Lyon: International Agency for Research on Cancer, 1986.

2 IARC Working Group on Evaluation of Cervical Cancer Screening Programmes Screning for squamous cervical cancer: duration of low risk fur grammes. Screning or squat (1)

3 Boyes DA, Morrison B, Knox EG, Draper GJ, Miller AB. A cohort study of cervical cancer screening in British Columbia. Clin Invest Med 1982;5:1-29. 4 Van Oortmarssen GJ, Habbema JDF. Epidemiological evidence for age dependent regression of pre-invasive cervical cancer. Br $\mathcal{J}$ Cancer 1991;64: 559-64.

5 Habbema JDF, Lubbe JThN, van Agt HME, van Ballegooijen M, Koopmanschap MA, van Oortmarssen GJ. The costs and effects of mass screening for cervical cancer. Rotterdam: Erasmus University, Department of Public Health and Social Medicine, 1988. (In Dutch.)

6 Gustafsson L, Adami HO. Natural history of cervical neoplasia: consistent results obtained by an identification technique. Br f Cancer 1989;60:132-41.

Van der Graaf Y. Screening for cervical cancer. The Nijmegen project. (PhD thesis.) Nijmegen: Katholieke Universiteit, 1987.

8 Cancer Registry of Norway. Survival of cancer patients. Cases diagnosed in Norway 1968-75. Oslo: Norwegian Cancer Registry, 1980.

9 Hakulinen T, Pukkala E, Hakama M, Lehtonen M, Saxen E, Teppo L. Survival of cancer patients in Finland 1953-1974. Ann Clin Res 1983;13 (suppl 31):50-2.

10 Koopmanschap MA, Lubbe JThN, van Oortmarssen GJ, van Agt HME, van Ballegooijen M, Habbema JDF. Economic aspects of cervical cancer screening. Soc Sci Med 1990;10:1081-7.

11 van Ballegooijen M, Koopmanschap MA, van Oortmarssen GJ, Habbema JDF, Lubbe JThN, van Agt HME. Diagnostic and treatment procedures induced by cervical cancer screening. Eur $\mathcal{J}$ Cancer 1990;26:941-5.

12 Elliot PM, Tatersall MHN, Coppleson M, Russell P, Wong F, Coates AS, et al. Changing character of cervical cancer in young women. $B M \mathcal{F} 1989 ; 298$ : 288-90.

(Accepted 16 fune 1992)

\section{Endocrine adverse effects of omeprazole}

\section{Marie Lindquist, I Ralph Edwards}

WHO Collaborating Centre for International Drug

Monitoring, Box 26 S-751 03 Uppsala, Sweden Marie Lindquist, senior pharmaceutical officer I Ralph Edwards, medical director

Correspondence to: Ms Lindquist.

BMF 1992;305:451-2
Omeprazole is thought to act to reduce gastric acid through specific binding to the parietal cell proton pump hydrogen ion potassium ATPase. Selectivity is further strengthened by the drug's basic nature causing it to accumulate in acid spaces, where it is activated. Both cimetidine and ranitidine have been reported to cause gynaecomastia and impotence, though, unlike cimetidine, ranitidine does not bind to androgen receptors. There have been two single case reports of gynaecomastia during treatment with omeprazole. ${ }^{12}$ We add further cases and also record cases of impotence related to omeprazole. All had been reported within the World Health Organisation's programme for international drug monitoring as cases, and in all cases causality seemed possible.

\section{Case histories}

The cases represent the total reported experience of these adverse drug reactions up to December 1991. There were 15 cases of impotence and 15 of gynaecomastia or breast enlargement.

Impotence-All cases of impotence were in men (mean age 52.6 years). They had been taking $20-40 \mathrm{mg}$ omeprazole daily for a mean of four days (cases in which exact dates were recorded only) before onset. Other drugs were not reported in eight cases and not used in three. The treated condition was mostly reflux oesophagitis (12 cases). Full details of patients are given in the table.

Gynaecomastia or breast enlargement-Gynaecomastia occurred in 13 men (mean, age of 56.8 years), and breast enlargement occurred in two women aged 41 and 77. The doses of omeprazole used were $20 \mathrm{mg}$ daily in most patients, $40 \mathrm{mg}$ daily in two men, $60 \mathrm{mg}$ daily in one man, and either unknown or intermittent in three patients, including one woman. The mean time to onset (known in 12 cases) was 2.9 months. Most of the patients had either gastric or duodenal ulceration, only three having oesophagitis. In three cases the diagnosis was not recorded. One patient had the Zollinger-Ellison syndrome. The table gives details of the patients.

\section{Comment}

There have been two single case reports of gynaecomastia in patients taking omeprazole but none of impotence. Both reports point out that the mechanism for the gynaecomastia is not apparent from the pharmacology of the drug. The cases we report are further evidence of the adverse reaction and come from eight different countries. Furthermore, they include the hitherto unrecorded adverse effect of impotence. Confounding due to other disease or other drugs 